\title{
Electronic Spectroscopy And Its Interpretation
}

\author{
Pramod Kumar Thakur \\ Central Department of Physics, Tribhuvan University, Kirtipur
}

\begin{abstract}
Electronic Spectroscopy relies on the quantized nature of energy states. At given enough energy, an electron can be excited from its initial ground state or initial excited state (hot band) and briefly exist in a higher energy excited state. Electronic transitions involve exciting an electron from one principle quantum state to another. Without incentive, an electron will not transition to a higher level.
\end{abstract}

Keywords: Photon energy, wave length, electron spin, spectral bands, bond length

\section{INTRODUCTION}

In the electronic spectroscopy, the electron is excited first from an initial low energy state to a higher state by absorbing photon energy from the spectrophotometer. If the wavelength of the incident beam has enough energy to promote an electron to a higher level, then we can detect this in the absorbance spectrum. Once in the excited state, the electron has higher potential energy and will relax back to a lower state by emitting photon energy. This is called fluorescence and can be detected in the spectrum as well.

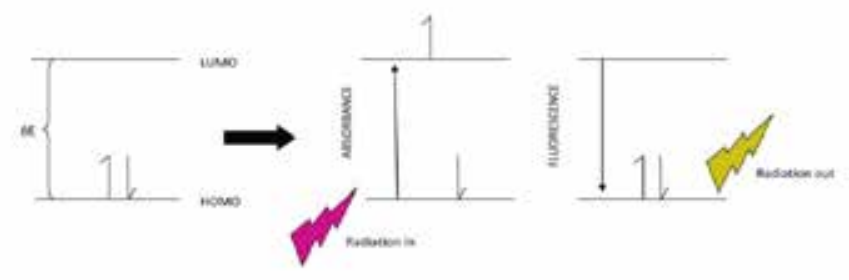

Embedded into the electronic states $(\mathrm{n}=1,2,3 \ldots)$ are vibrational levels $(\mathrm{v}=1,2,3 \ldots)$ and within these are rotational energy levels $(\mathrm{j}=1,2,3 \ldots)$. Often, during electronic transitions, the initial state may have the electron in a level that is excited for both vibration and rotation. In other words, $n=0, v$ does not $=0$ and $r$ does not $=0$. This can be true for the ground state and the excited state. In addition, due to the Frank Condon Factor, which describes the overlap between vibrational states of two electronic states, there may be visible vibrational bands within the absorption bands.

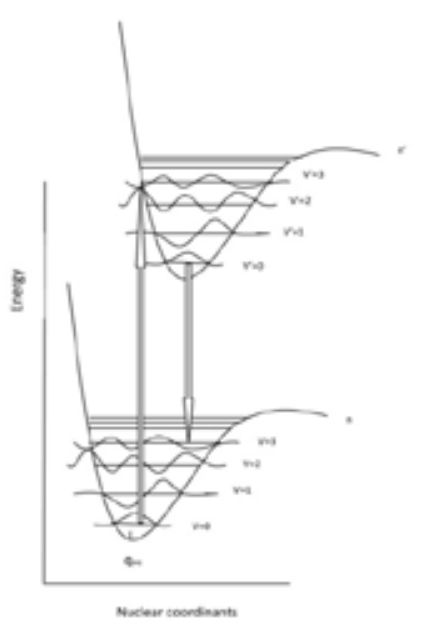

When interpreting the absorbance and fluorescence spectra of a given molecule, compound, material, or an elemental material, understanding the possible electronic transitions is crucial. Assigning the peaks in the absorption spectrum can become easier when considering which transitions are allowed by symmetry, electron spin, or vibronic coupling. Knowing the degree of allowedness, one can estimate the intensity of the transition, and the extinction coefficient associated with that transition. These guidelines are a few examples of the selection rules employed for interpreting the origin of spectral bands. Only a complete model of molecular energy diagrams for the species under investigation can make clear the possible electronic transitions.

The following section will discuss the interpretation of electronic absorption spectra given the nature of the chemical species being studied. This includes 
an understanding of the molecular or elemental electronic state symmetries, spin multiplicities, and forbidden and allowed transitions of a given species. As the light passes through the monochrometer of the spectrophotometer, it hits the sample with some wavelength and corresponding energy. The ratio of the initial intensity of this light and the final intensity after passing through the sample is measured and recorded as absorbance (Abs). When absorbance is measured at different wavelengths, an absorbance spectrum vs wavelength can be obtained. This spectra reveals the wavelengths of light that are absorbed by the chemical specie, and is specific for each different chemical. Many electronic transitions can be visible in the spectrum if the energy of the incident light matches or surpasses the quantum of energy separating the ground state and that particular excited state. An example of an absorbance spectrum is given below.
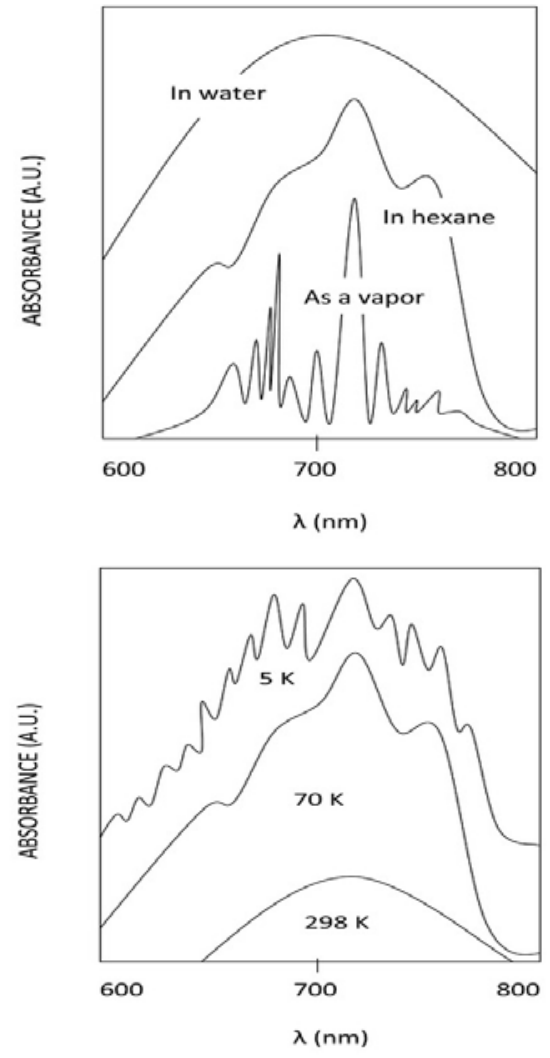

\section{EFFECTS OF TEMPERATURE}

Here we can see the effect of temperature and also the effect of solvents on the clarity of the spectrum. We can see from these anonymous compounds that decreasing the temperature allows the vibrational fine structure to emerge. These vibrational bands embedded within the electronic bands represent the transitions from $\mathrm{v}=\mathrm{n}$ to $\mathrm{v}^{\prime}=\mathrm{n}$. Generally, the $\mathrm{v}=0$ to $\mathrm{v}^{\prime}=0$ transition is the one with the lowest frequency. From there, increasing energy, the transitions can be from $\mathrm{v}=0$ to $\mathrm{v}^{\prime}=\mathrm{n}$, where $\mathrm{n}=1,2,3 \ldots$

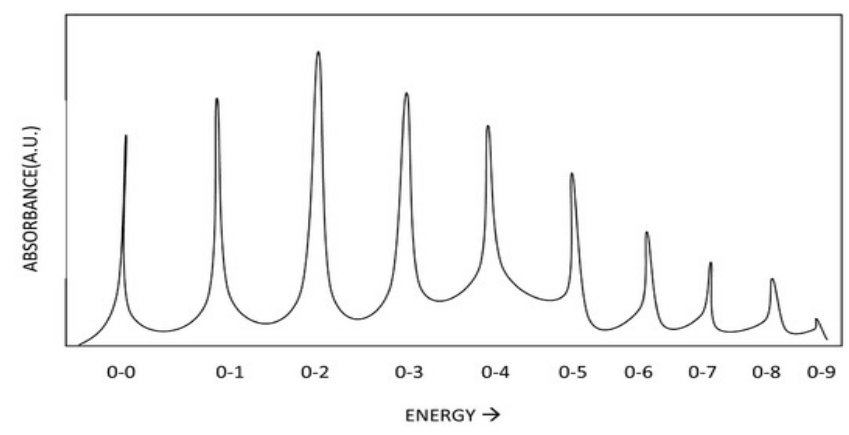

On the left, we see the electronic absorption of diatomic molecule. The bands represent the jumping of the electron from the ground electronic state/ground vibrational state $\mathrm{n}=0 / \mathrm{v}=0$ to the excited electronic state/ground vibrational state $\mathrm{n}=1 / \mathrm{v}=0$. This is denoted as $0-0$ and is defined as the lowest energy transition. The other transitions involve an electronic transition in combination with vibrational transitions within the excited electronic state. In other words, the bands labeled $0-1,0-2, \ldots$ etc represent the electrons that travel higher in energy than the purely electronic transition (00 ). This introduces the possibility of vibrational information within the electronic spectrum. In fact, due to the Frank Condon overlap of the ground state and excited state, the 0-2 transition is the most probable of all of the transitions. We can see this in the spectra as the

$0-2$ has the greatest absorbance. If the potential energy wells of the ground and excited states were perfectly eclipsed on top of one another, then the $0-0$ transition would be the most probable transition with the highest intensity band. This however, as we have seen, is not often the case. In deed the equilibrium bond length tends to increase as the energy of the state increases usually due to antibonding nature of the state increasing the intramolecular bond length. 


\section{HOT BANDS}

Some transitions are forbidden by symmetry and do not appear in the absorption spectrum. If the symmetries are correct, then another state besides the ground state can be used to make the otherwise forbidden transition possible. This is accomplished by hot bands, meaning the electrons in the ground state are heated to a higher energy level that has a different symmetry. When the transition moment integral is solved with the new hot ground state, then the direct product of the symmetries may contain the totally symmetric representation.Knowing whether a transition will be allowed by symmetry is an essential component to interpreting the spectrum. If the transition is allowed, then it should be visible with a large extinction coefficient. If it is forbidden, then it should only appear as a weak band if it is allowed by vibronic coupling. In addition to this, a transition can also be spin forbidden. The examples below of excited state symmetries, give an indication of what spin forbidden means:

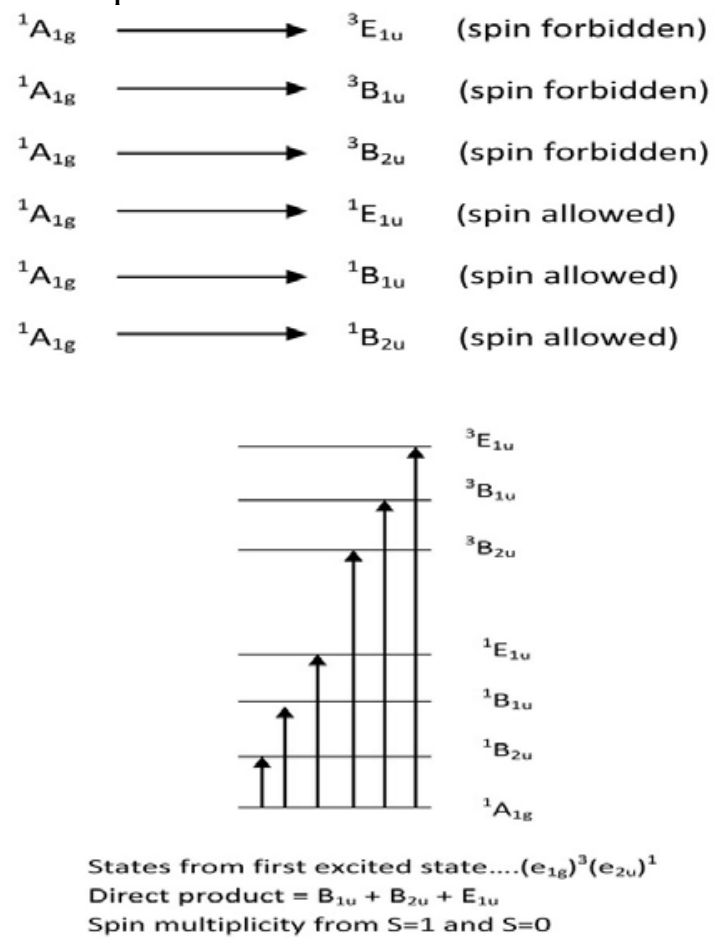

These states are derived from the electron configuration of benzene. Once we have the molecular orbital energy diagram for benzene, we can assign symmetries to each orbital arrangement of the ground state. From here, we can excite an electron from the Highest Occupied Molecular Orbital (HOMO) to the Lowest Unoccupied Molecular Orbital (LUMO). This is the lowest energy transition. Other transitions include moving the electron above the LUMO to higher energy molecular orbitals. To solve for the identity of the symmetry of the excited state, one can take the direct product of the HOMO symmetry and the excited MO symmetry. This give a letter (A, B, E..) an the subscript $(1 \mathrm{u}, 2 \mathrm{u}, 1 \mathrm{~g} . .$.$) . The superscript$ is the spin multiplicity, and from single electron transitions, the spin multiplicity is $2 \mathrm{~S}+1=\mathrm{M}$, where $\mathrm{S}=1$ with two unpaired electrons having the same spin and $S=0$ when the excited electron flips its spin so that the two electrons have opposite spin. This gives $\mathrm{M}=1$ and $\mathrm{M}=3$ for benzene above. From the above, we have three transitions that are spin allowed and three that are spin forbidden.

\section{FLUORESCENCE}

Now that we have discussed the nature of absorption involving an electron absorbing photon energy to be excited to a higher energy level, now we can discuss what happens to that excited electron. Due to its higher potential energy, the electron will relax back to its initial ground state, and in the process, emit electromagnetic radiation. The energy gap between the excited state and the state to which the electron falls determines the wavelength of light that will be emitted. This process is called fluorescence.Given the following diagram, one can see that vibrational relaxation occurs in the excited electronic state such that the electronic relaxation occurs from the ground vibrational state of the excited electronic state. This causes lower energy electronic relaxations than the previous energy of absorption. 


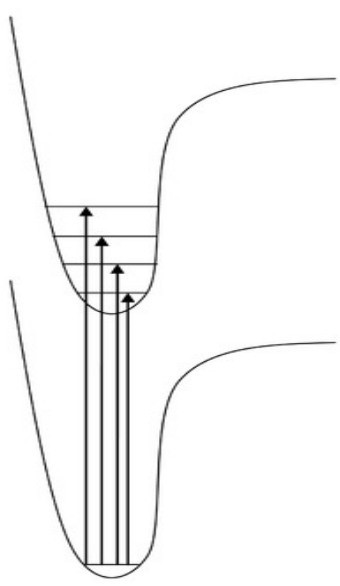

Absorption

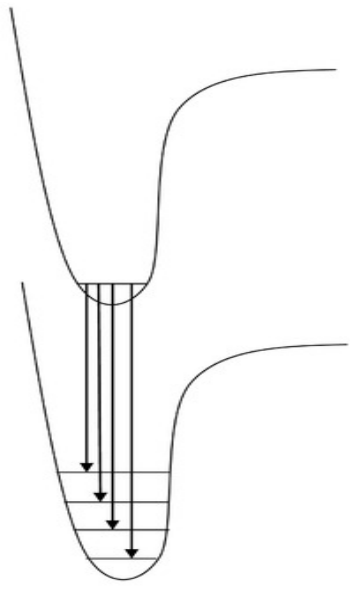

Fluorescence
Due to vibrational relaxation in the excited state, the electron tends to relax only from the $v^{\prime}=0$ ground state vibrational level. This gives emission transitions of lower energy and consequently, longer wavelength than absorption. When obtaining fluorescence, we have to block out the transmitted light and only focus on the light being emitted from the sample, so the detector is usually 90 degrees from the incident light. Because of this emission spectra are generally obtained separately from the absorption spectra; however, they can be plotted on the same graph as shown.

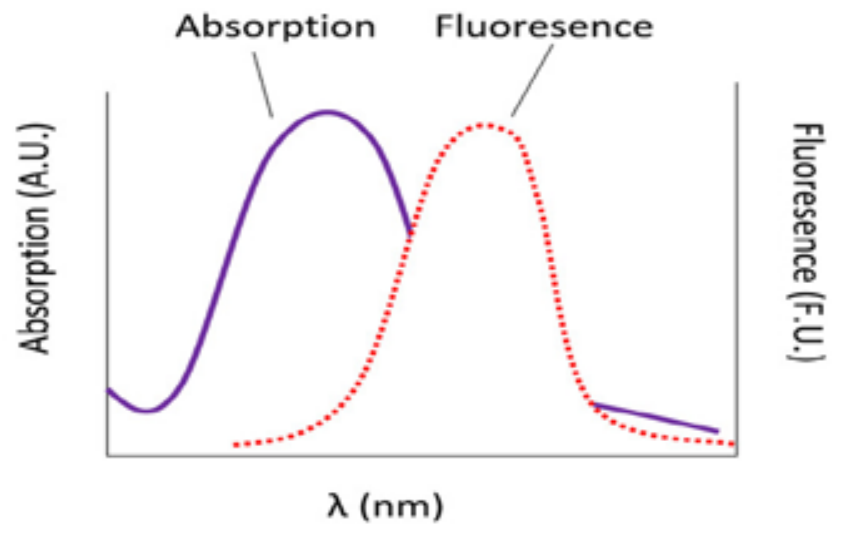

\section{CONCLUSION:}

In the vibrational fine structure that can be seen in the absorption spectrum gives some indication of the degree of Frank Condon overlap between electronic states. At higher temperature, the vibrational transitions become averaged in the spectrum due to the presence of vibrational hot bands and Fermi Resonance, and with this, the vibrational fine structure is lost at this temperature.Also at the absorption transitions by default involve a greater energy change than the emission transitions and the fluorescence peak follows the absorption peak according to the spectrum.

\section{REFERENCES}

1. Harris, Daniel; Bertolucci, Michael. Symmetry and Spectroscopy. An Introduction to Vibrational and Electronic Spectroscopy. Dover Publications, Inc., New York, 1989.

2. Condon,E. Electron Transition of Diatomic molecule.(Phy.Rev) ,1928.

3. Bemath,Peter F. Spectra of Atoms and Molecules.(Physical Chemistry) ,Oxford,1995. 